\title{
A Stochastic Geometry Model for Multi-hop Highway Vehicular Communication
}

\author{
Muhammad Junaid Farooq, Student Member, IEEE, Hesham ElSawy, Member, IEEE, and \\ Mohamed-Slim Alouini, Fellow, IEEE
}

\begin{abstract}
Carrier sense multiple access (CSMA) protocol is standardized for vehicular communication to ensure a distributed and efficient communication between vehicles. However, several vehicular applications require efficient multi-hop information dissemination. This paper exploits stochastic geometry to develop a tractable and accurate modeling framework to characterize the multi-hop transmissions for vehicular networks in a multi-lane highway setup. In particular, we study the tradeoffs between per-hop packet forward progress, per-hop transmission success probability, and spatial frequency reuse (SFR) efficiency imposed by different packet forwarding schemes, namely, most forward with fixed radius (MFR), the nearest with forward progress (NFP), and the random with forward progress (RFP). We also define a new performance metric, denoted as the aggregate packet progress (APP), which is a dimensionless quantity that captures the aforementioned tradeoffs. To this end, the developed model reveals the interplay between the spectrum sensing threshold $\left(\rho_{t h}\right)$ of the CSMA protocol and the packet forwarding scheme. Our results show that, in contrary to ALOHA networks which always favor NFP, MFR may achieve the highest APP in CSMA networks if $\rho_{t h}$ is properly chosen.
\end{abstract}

\section{INTRODUCTION}

The world wide interest to develop intelligent transportation systems (ITS), that improve the efficiency and safety of transportation, increases the research focus on vehicular networking. While ITS cover all forms of transportation, including road, rail, air and water transportation, communication for road networks is of special interest due to its uncoordinated and less controlled nature when compared to the other transportation systems. While planes, trains, and ships are usually driven by highly trained personnel, cars are driven by public varying in their skills, which raises serious safety issues for road transportation. For instance, according to the US census bureau, the US has a round figure of 10 million road accident per year with an average of $1.5 \%$ fatality rate [?]. The US national highway traffic safety administration (NHTSA) reports that an average of 90 deaths and more than 6000 injuries occur every day, across the US, due to car accidents [?]. Hence, there is a high demand to incorporate an automated and intelligent system to compensate for the human error, which is considered the major cause for accidents. In this

Muhammad Junaid Farooq, Hesham ElSawy and Mohamed-Slim Alouini are with the Electrical Engineering Program, Computer, Electrical and Mathematical Sciences and Engineering (CEMSE) Division, King Abdullah University of Science and Technology (KAUST), Thuwal, Makkah Province, Saudi Arabia. (E-mails: \{muhammadjunaid.farooq, hesham.elsawy, slim.alouini\}@kaust.edu.sa).

Research reported in this publication was supported by the King Abdullah University of Science and Technology (KAUST). context, vehicles can communicate with each other to alert drivers, or even take automated actions, to avoid collisions and save lives. Vehicular communication can also offer potential gains from an economical perspective. A recent report by The Economist estimated the total cost of traffic jams across four countries (US, UK, Germany, and France) to be 200 billion dollars [?]. In this case, vehicular networking can be exploited to improve navigation systems and alleviate traffic jams, i.e., to develop real time and context-aware navigation systems that collect real-time traffic information, via the vehicular network, to take appropriate navigation decisions [?].

A typical vehicular communication system consists of three different links or modes of operation, namely, vehicle-tovehicle $(\mathrm{V} 2 \mathrm{~V})$, vehicle-to-roadside (V2R), and roadside-toroadside. The $\mathrm{V} 2 \mathrm{~V}$ communication, also referred to as intervehicle communication (IVC) [?], is an enabling technology for a variety of applications such as adaptive traffic control, coordinated braking, emergency messaging, peer-topeer networking for infotainment services, and automatic toll collection. Further, IVC communication is preferred over V2R in high mobility environments as well as for applications with strict delay constraint such as the safety based dedicated short-range communications (DSRC) [?]. According to the IEEE standard, IVC is coordinated via carrier sense multiple access (CSMA) medium access control (MAC) protocol. CSMA is a contention based spectrum access technique that is controlled by means of random backoff counters. That is, each transmitter, that intends to transmit a packet, generates a random backoff timer which is decremented if the channel is sensed idle and freezed if the channel is sensed busy. Only transmitters with elapsed backoff timers can transmit. Hence, a transmission is only initiated in an idle channel state to avoid packet collisions. The idle/busy state of the channel is determined by the carrier sensing threshold $\left(\rho_{t h}\right)$ [?], which is a vital design parameter for the CSMA protocol. The sensing threshold imposes a crucial tradeoff between the transmission success probability or simply the success probability, denoted by $\mathcal{P}$, and the spatial frequency reuse (SFR) efficiency. A lower sensing threshold enforces larger distances between concurrent transmitters, which decreases interference and increases the success probability, on the expense of degrading the SFR, and vice versa. Hence, the sensing threshold $\left(\rho_{t h}\right)$ should be carefully tuned to balance the tradeoff between the success probability and SFR efficiency, thus achieving efficient local communication.

Several vehicular applications require going beyond the local communication and efficiently disseminating information 
across the roads via multi-hops, as shown in Fig ??. For instance, accidents on highways can be immediately reported to the nearest ambulance or emergency center using the multi-hop vehicular network. Also in the case of highway congestion, it is important to disseminate the information far enough so that other vehicles can take appropriate navigational decisions and change the selected trajectory to alleviate the congested sector. Therefore, packets need to be efficiently propagated from one hop to another until they arrive at the destination. There are different packet forwarding schemes, that impose a tradeoff between packet forward progress (FP) and per-hop success probability. In particular, we focus on the most forward with fixed radius (MFR), the nearest with forward progress (NFP), and the random with forward progress (RFP) strategies. As it is clear from the protocol names, the MFR favors relaying the packets to a distant vehicle to maximize the forward progress on the expense of lower per-hop success probability. On the contrary, the NFP favors communicating with the nearest vehicle to attain a high per-hop success probability on the expense of low forward progress. The RFP achieves a midpoint performance between the MFR and the NFP. It is worth mentioning that there is an interaction between the carrier sensing threshold and the packet forwarding protocols. For instance, to have an acceptable success probability, the MFR requires more conservative $\rho_{t h}$ due to the large hop distance as compared to the NFP which can sustain an aggressive $\rho_{t h}$ due to the smaller hop distance. This directly has an impact on the end-to-end transmission delay or equivalently the throughput since the $M F R$ requires lesser number of transmissions to reach a particular destination as compared to the NFP which requires more number of transmissions. Note that, an efficient packet forwarding scheme should achieve the best balance between the success probability, packet FP, and the SFR efficiency imposed by both the forwarding scheme and the CSMA parameter $\rho_{t h}$, while maintaining a particular end-toend transmission delay.

In this paper, we focus on the IVC aspects in vehicular networks in a multi-lane highway scenario and study three different packet forwarding strategies. In particular, we develop a novel analytical framework to model CSMA coordinated IVC in a highway setup using concepts from stochastic geometry and point process theory. We obtain simple yet accurate expressions for the per-hop success probability as well as the forward progress. Our developed model captures the tradeoffs among the success probability, forward progress, and the SFR efficiency of each forwarding scheme. In order to unify these three performance metrics, we define the aggregate packet progress (APP).

Our results show that $N F P$ achieves the highest APP in networks that require high link quality ${ }^{1}$ per transmission but with relaxed delay constraint. On the contrary, $M F R$ achieves the highest APP in networks with tight delay constraint and relaxed link quality. It is worth noting that ALOHA networks always favor NFP due to the high interference levels in the network [?], [?]. In contrast, sufficient interference protection

\footnotetext{
${ }^{1}$ The required link quality is quantified by the signal-to-interference-plusnoise-ratio required for correct signal reception.
}

can be achieved in CSMA networks by proper manipulation of the sensing threshold, which makes the MFR strategy preferable is some cases. The main contributions of this paper are summarized as:

1) We develop a novel tractable framework to model intervehicle communication in a multi-lane highway network.

2) We compare between various abstraction models used for highway vehicular networks and show that the widely used line abstraction model does not accurately model the success probability for wide highways.

3) We study different packet dissemination strategies for multi-hop communication in CSMA based IVC and compare their performance under different traffic conditions.

4) We define the aggregate packet progress (APP) metric which captures the tradeoff between success probability, forward progress, and spatial frequency reuse efficiency.

5) We show that we can select a packet forwarding strategy with an appropriate carrier sensing threshold to maximize the APP under specific QoS constraints.

\section{RELATED WORK}

Balancing the tradeoff between the success probability and the spatial frequency reuse is a major challenge in wireless ad hoc networks. In the literature, different metrics are used to capture this fundamental tradeoff. For instance, transmission capacity metric is used in [?], spatial throughput is used in [?], and spatial reuse is used in [?] to quantify the number of successful transmissions per unit area. These metrics are mainly used to gain insights into the system behavior and to optimize the network performance. In addition to the success probability and the spatial frequency reuse, the effect of the forward progress in multi-hop ad hoc networks is incorporated into the analysis in [?], [?]. However, [?], [?] consider conventional 2-D ad hoc networks. Note that vehicular ad hoc networks (VANETs) differ from general ad hoc networks by having their own characteristics (e.g., geographical limitation to roads) that should be incorporated into the modeling framework.

In the context of VANETs, the authors in [?] and [?] develop an analytical model to maximize the network throughpu2 However, the presented models are based on ALOHA spectrum access protocol. Although CSMA based IEEE 802.11p protocol is the standardized access protocol for vehicular communication, it is shown in [?] that the performance of the IEEE 802.11p approaches the performance of ALOHA protocol at sufficiently high node densities due to backoff timer ties 3 . The ALOHA based network models in [?], [?], [?] do not aptly capture the network performance when CSMA protocol operation is intact. Note that alleviating the backoff timer ties problem in the IEEE 802.11p to achieve an intact CSMA spectrum access is viable by means of adaptive contention window size techniques [?], [?].

\footnotetext{
${ }^{2}$ Note that the throughput is directly linked to the forward progress in VANETs [?]

${ }^{3}$ IEEE 802.11 p is similar to the IEEE 802.11 protocol except for the discrete backoff timer values which reduces the contention time on the expense of having flawed CSMA operation at high node density
} 


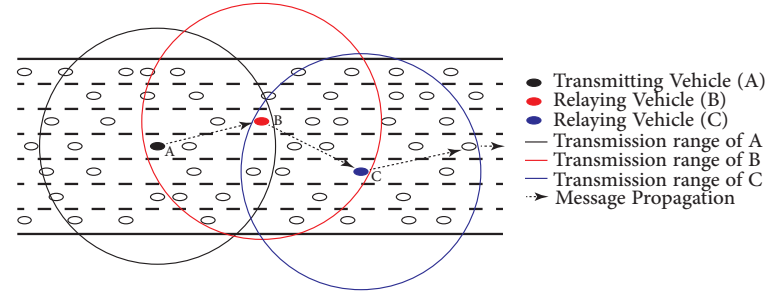

Fig. 1: Message propagation in multi-lane highways using the $M F R$ strategy. A test transmitting vehicle A forwards the packet to a vehicle $B$ that results in the most forward progress inside its transmission range. This process continues until the packet reaches its destination.

VANETs with an intact CSMA spectrum access have been studied in [?], [?], [?], [?]. In [?], the maximum possible spatial reuse in a VANET is investigated. The authors in [?], [?], [?] conduct performance and reliability analysis for vehicular networks. Most of the related works on VANETS using CSMA protocol are based on the the single line abstraction (SLA) model. The SLA model combines multiple highway lanes into a single lane with the aggregated traffic, which may not be accurate for wide highways as well as for high traffic intensity [?]. The models developed for CSMA based VANETs in the literature are mostly limited to local vehicular communication.

This paper focuses on VANETs with intact CSMA spectrum access and develop a novel analytical paradigm for multi-hop communication in multi-lane highway. The developed model does not rely on the SLA model and captures the effect of the number of traffic lanes and the inter-lane separation. The paper also studies the tradeoff imposed by the CSMA sensing threshold and the packet forwarding strategy on success probability, packet forward progress and SFR efficiency. To this end, the developed analytical framework is used to select the forwarding strategy and the carrier sensing threshold to optimize the performance tradeoffs.

\section{System MODEL}

In this section, we describe the network model, discuss the considered transmission strategies, and explain the methodology of analysis.

\section{A. Network Model}

We consider a multi-lane highway network constituted from $N$ parallel lanes separated by a fixed distance $d$. It is assumed that each traffic lane has an infinite length and is populated by vehicles according to an independent and homogeneous 1D Poisson point process (PPP) of intensity $\lambda$ vehicles $/ \mathrm{km}$ per lane, i.e. $\Phi_{i}=\left\{v_{i j} ; j=0,1,2, \ldots\right\}$, where $i=1, \ldots, N$ is the lane index and $v_{i j}$ represents the location of the $j^{\text {th }}$ vehicle on the $i^{t h}$ traffic lane. Note that for ease of analysis, we assume a homogeneous traffic in which each lane has the same intensity of traffic. A snapshot of the network model is shown in Fig. ??. For a test vehicle under analysis, there are $N_{R}$ traffic lanes on its right side and $N_{L}$ traffic lanes on its left side where $N=N_{R}+N_{L}+1$. The lane containing the vehicle under analysis is referred to as the central lane. Each vehicle transmits with a fixed power $P$, or equivalently has a fixed transmission range $R_{t}$. The signal power decays with the distance according to the power law propagation model with a path-loss exponent $\eta>1$. Beside path loss attenuation, the signal power experiences fading which is assumed to be exponential with mean of $1 / \mu$. Therefore the power received from a transmitter located at $v_{i}$ to the receiver at $v_{j}$ is $P h_{i j}\left\|v_{i}-v_{j}\right\|^{-\eta}$, where $h_{i j}$ represents the channel fading between $v_{i}$ and $v_{j}$ and $\|$.$\| represents the Euclidean$ norm. We assume that during each time slot, the network topology remains the same and is independent of other time slots. This is a reasonable assumption since the speed of packet transmission including the delays due to backoff is much faster than the speed of vehicles. This is formally stated as:

Assumption 1: We assume that the network topology remains constant for the duration of each time slot and changes independently from one time slot to the other.

Remark 1: According to [?], the maximum average wait time before transmission is estimated to be $264 \mu \mathrm{s}$ and the total packet delivery time is in the order of milliseconds. Therefore on an $80 \mathrm{~km} / \mathrm{h}$ highway, a vehicle is displaced by much less than $1 \mathrm{~m}$ during a single transmission. This makes it reasonable to ignore the effect of mobility during contention and packet transmission time.

We consider a signal capture model, which assumes that a packet can be correctly decoded by the receiver iff the signal-to-interference-plus-noise-ratio (SINR) exceeds a target threshold $T$. All vehicles contend for channel access according to the slotted CSMA protocol. For the CSMA contention process, we have the following assumption:

Assumption 2: We assume that no two vehicles in the same contention domain have the same backoff counter value and hence the CSMA behaviour of IEEE 802.11p is intact.

Remark 2: The basic operation of the IEEE 802.11p is similar to the conventional operation of the CSMA protocol except for the higher collision probability within the contention domain due to discrete backoff counter values [?]. For this reason, several efforts have been invested to adapt the contention window (i.e., backoff timer size) to alleviate this problem [?], [?]. Since the framework proposed in this paper focuses on the spatial domain uncertainties and abstracts time dynamics, we can assume that the contention domain collision problem is solved by one of the techniques proposed in the literature (e.g., [?], [?]).

The carrier sensing threshold $\rho_{t h}$ of the CSMA protocol is a system wide design parameter used by all vehicles. From a geometric perspective, $\rho_{t h}$ can be directly translated to a sensing range, also denoted as the contention domain, represented by $R_{s}=\left(\frac{P}{\rho_{t h}}\right)^{1 / \eta}$. Note that the transmission range or neighbourhood domain is similarly expressed as $R_{t}=\left(\frac{P}{\rho_{\text {min }}}\right)^{1 / \eta}$ where $\rho_{\min }$ is the receiver sensitivity. The sensing range affects the spatial frequency reuse because the CSMA protocol does not allow the same channel to be used by two vehicles in the same contention domain, where the contention domain of the $j^{t h}$ vehicle on the $i^{t h}$ lane $\left(v_{i j}\right)$ is defined as $\mathcal{N}_{v_{i j}}=\left\{v_{m n}: P\left\|v_{i j}-v_{m n}\right\|^{-\eta} \geq \rho_{t h}, \forall m, n\right\}$. If $v_{i j}$ is in the contention domain of $v_{m n}$, then using the 
assumption of reciprocity ${ }^{4}$ in wireless channels, the converse is also true. Thus, for notational brevity, we drop the subscripts of $v_{i j}$, and hereafter refer to the contention domain of a typical vehicle as $\mathcal{N}$. The average number of vehicles in the contention domain of a vehicle with $N_{R}$ traffic lanes on its right side and $N_{L}$ traffic lanes on its left is given by:

$$
\mathbb{E}[|\mathcal{N}|]=2 \lambda\left(R_{s}+\sum_{i=1}^{N_{R}} \sqrt{R_{s}^{2}-(i d)^{2}}+\sum_{j=1}^{N_{L}} \sqrt{R_{s}^{2}-(j d)^{2}}\right),
$$

where |.| represents the set cardinality. In a similar manner, we can define the transmission neighbourhoo 5 of a typical node as $\mathbf{N}_{v_{i j}}=\left\{v_{m n}: P\left\|v_{i j}-v_{m n}\right\|^{-\eta} \geq \rho_{m i n}, \forall m, n\right\}$. The average number of neighbours of a transmitting vehicle with transmission range $R_{t}$ and with $N_{R}$ and $N_{L}$ traffic lanes on its right and left side respectively is, therefore:

$\mathbb{E}[|\mathbf{N}|]=2 \lambda\left(R_{t}+\sum_{i=1}^{N_{R}} \sqrt{R_{t}^{2}-(i d)^{2}}+\sum_{j=1}^{N_{L}} \sqrt{R_{t}^{2}-(j d)^{2}}\right)$.

\section{B. Packet Forwarding Strategies}

From a transmitter's perspective, there are several strategies to select the relaying vehicle from its neighbors. The most simple strategies are to transmit to either the nearest node, the farthest node, or to a random node inside the transmission range [?]. We assume that the packet's destination is located far enough such that the progress can be defined as the horizontal distance moved along the highway towards the receiver. We only allow forward progress in which the message direction is known (e.g., the location of the nearest emergency center in case of accidents). The different transmission strategies are described as follows:

1) Most Forward with Fixed Radius (MFR): In this strategy, a typical transmitting node forwards the packet to the neighbour that results in the most forward progress within its transmission range. MFR maximizes the forward progress on the expense of low success probability due to the imposed large transmitter-receiver spacing. Note that $M F R$ requires a conservative sensing threshold (i.e., $\zeta \triangleq R_{s} / R_{t} \geq 1$ ) such that the sensing range can provide sufficient interference protection for the receiver. Note also that a conservative sensing threshold implies low spatial frequency reuse.

2) Nearest with Forward Progress (NFP): In this strategy, a typical transmitting node forwards the packet to its closest neighbour. This would increase the per-hop success probability on the expense of minimizing the forward progress. However, the NFP strategy can sustain an aggressive sensing threshold (i.e., $\zeta \triangleq R_{s} / R_{t} \ll 1$ ) due to the small link distance (i.e., short transmitter-receiver separation) which improves the desired signal received power.

\footnotetext{
${ }^{4}$ The wireless channel between vehicles is assumed to be symmetric w.r.t. the roles of transmitter and receiver.

${ }^{5}$ vehicles inside the transmission range of each other are considered to be neighbours.
}

$x$ - Vehicle Location

(a) Single Lane Abstraction Model

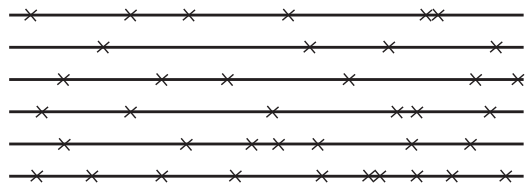

(b) Multi-lane model

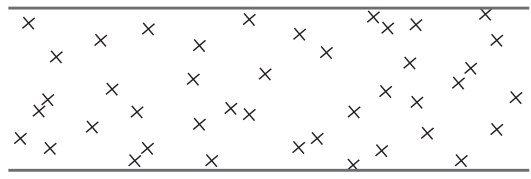

(c) 2-D PPP model

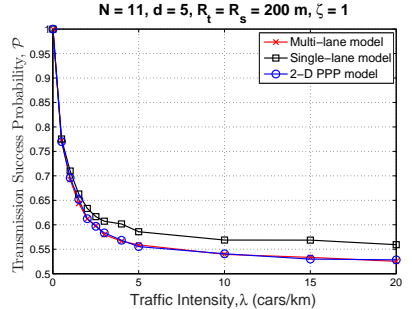

(d) Model comparison for $N=11$

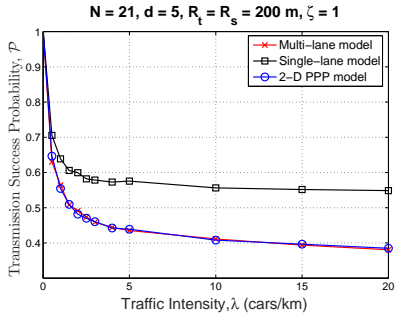

(e) Model comparison for $N=21$
Fig. 2: Modeling approaches for multi-lane highways. The simulations in (d) \& (e) show that the single-lane model is not an accurate model for multi-lane highways. The deviation of the singlelane model from the multi-lane and 2D-PPP model increases as the highways become wider.

3) Random with Forward Progress (RFP): In this strategy, every potential receiver, that results in forward packet progress, has an equal probability of being selected by the protocol. This strategy achieves a midpoint performance between the $M F R$ and the NFP.

\section{Modeling Approaches}

There are three main approaches to model vehicles on a multi-lane highway as illustrated in Fig. ??. The first approach, which is widely used in literature, is known as the Single Lane Abstraction (SLA) model or simply the line abstraction model shown in Fig. ?? in which all the traffic lanes are merged into a single lane with the aggregated traffic intensity. This greatly simplifies the analysis. However, it has been shown in [?] that the line abstraction model cannot accurately characterize the performance of highway vehicular networks when the highway is significantly wide and under high traffic intensity. The second approach is to consider that the traffic is restricted into individual lanes separated by a fixed inter-lane distance, as illustrated in Fig. ??. The third approach models the highway traffic using a 2-D PPP restricted to the area covered by the highway, as illustrated in Fig. ??. Compared 
to the second approach, the third approach gives one more degree of freedom on the location of the vehicles. However it is not tractable. A simulation study comparing the results of the three approaches under the RFP strategy (see Fig. ?? and ??) shows that the multi-lane approach and the 2-D PPP approach give approximately the same results while the SLA model overestimates the probability of successful transmission. The same conclusion is drawn for the MFR and NFP strategy. Therefore, we use the second approach to conduct the analysis in this paper.

\section{Methodology of Analysis}

Our objective is to study the tradeoff between the success probability, forward packet progress, and the spatial frequency reuse efficiency imposed by the forwarding schemes and the CSMA parameter $\rho_{t h}$. Hence, our main performance metrics are per-hop success probability, the forward progress, and the aggregate packet progress. Before conducting the main analysis, three auxiliary parameters need to be calculated, namely, the transmitter-receiver distance distribution, the intensity of concurrent transmitters, and the Laplace transform (LT) of the probability density function (pdf) of the aggregate interference. While the transmitter-receiver distance distribution is necessary to characterize the desired signal power at the test receiver, the intensity of concurrent transmitters is required to characterize the interference power at the test receiver. Note that the interference power is characterized via its LT.

\section{SINR CHARACTERIZATION}

Due to the shared nature of the wireless medium, SINR is a major performance metric. The per-hop transmission is considered successful (i.e., correctly decoded by the receiver) if the SINR is above a predefined threshold. The SINR can be written as:

$$
\operatorname{SINR}_{\chi}=\frac{P h_{o} r_{\chi}^{-\eta}}{\kappa+\underbrace{\sum_{i=1}^{N} \sum_{v_{i j} \in \tilde{\Phi}_{i} \backslash v_{0}} P h_{i j}\left\|v_{i j}\right\|^{-\eta}}_{I_{a g g}}},
$$

where $\chi \in\{M, N, R\}$ is the packet forwarding mode, where $M, N$, and $R$ denote the $M F R$, the $N F P$, and $R F P$ respectively. The distance $r_{\chi}$ denotes the transmitter-receiver separation when using $\chi$ forwarding scheme, the set $\tilde{\Phi}_{i} \backslash v_{0} \subseteq \Phi_{i}$ denotes the set of active transmitters, excluding the intended transmitter $v_{o}$, which is obtained after dependent thinning of the original PPP due to the CSMA contention. $v_{i j} \in \mathbb{R}^{2}$ is the location of the $j^{\text {th }}$ interfering vehicle on the $i^{\text {th }}$ traffic lane. Finally, $h_{o}$ and $h_{i j}$ are i.i.d. random variables representing the channel fading between the test transmitting vehicle and the selected receiver and between the receiver and $i j^{\text {th }}$ interfering vehicle respectively, and $\kappa$ is the noise variance. From (??), it can directly observed that SINR is a random variable that includes several uncertainties which are, the vehicle locations, the channel gains, the CSMA contention based access, as well as the transmitter-receiver separation. The complementary cumulative distribution function (ccdf) of the SINR defines the per-hop success probability, which can be calculated as follows (see equation (5) in [?]):

$$
\begin{aligned}
\mathcal{P}_{\chi} & =\mathbb{P}\left\{S I N R_{\chi}>T\right\}, \\
& =\int_{r} \exp \left\{-\frac{T \mu \kappa r^{\eta}}{P}\right\} \mathcal{L}_{I_{a g g}}\left(\frac{T \mu r^{\eta}}{P}\right) f_{r_{\chi}}(r) d r,
\end{aligned}
$$

where $\mathcal{L}_{I_{\text {agg }}}($.$) is the LT of I_{a g} \rrbracket^{6}$ Equation (??) shows the auxiliary metrics that are required to evaluate the per-hop success probability, namely, the transmitter-receiver separation pdf $f_{r_{\chi}}($.$) and the LT of the aggregate interference I_{a g g}$. Note the the LT of the aggregate interference is a function of the intensity of concurrent transmitters (or interferers).

\section{A. Distance Distribution}

Since the receiver selection is based on the underlying packet forwarding protocol, the transmitter-receiver distance distribution is different for $M F R, N F P$, and $R F P$. For the $M F R$ the transmitter-receiver distance distribution is obtained via the following lemma:

Lemma 1: The distance $r_{M}$ between the transmitter and the receiver in the $M F R$ strategy is distributed as follows:

$$
\begin{aligned}
& f_{r_{M}}(r)= \\
& \left\{\begin{array}{cc}
-\frac{\lambda e^{-\lambda \mathbf{A}(r)}}{N\left(1-e^{\mathbb{E}[|\mathbf{N}|] / 2}\right)} \mathbf{A}^{\prime}(r), & 0<r \leq d, \\
-\frac{\lambda e^{-\lambda \mathbf{A}(r)}}{N\left(1-e^{\mathbb{E}[|\mathbf{N}|] / 2}\right)} \mathbf{A}^{\prime}(r)-\frac{2 \lambda \exp \left(-\lambda \mathbf{A}\left(\sqrt{r^{2}-d^{2}}\right)\right)}{N\left(1-e^{\mathbb{E}[|\mathbf{N}|] / 2}\right)} \mathbf{A}^{\prime}\left(\sqrt{r^{2}-d^{2}}\right), & d<r \leq 2 d, \\
\vdots & d< \\
-\frac{\lambda e^{-\lambda \mathbf{A}(r)}}{N\left(1-e^{\mathbb{E}[|\mathbf{N}| / 2]}\right)} \mathbf{A}^{\prime}(r)- & \\
\sum_{i=1}^{N_{R}} \frac{\lambda \exp \left(-\lambda_{l} \mathbf{A}\left(\sqrt{r^{2}-(i d)^{2}}\right)\right)}{N\left(1-e^{\mathbb{E}[|\mathbf{N}|] / 2}\right)} \mathbf{A}^{\prime}\left(\sqrt{r^{2}-(i d)^{2}}\right)- & \\
\sum_{i=1}^{N_{L}} \frac{\lambda \exp \left(-\lambda_{l} \mathbf{A}\left(\sqrt{r^{2}-(i d)^{2}}\right)\right)}{N\left(1-e^{\mathbb{E}[|\mathbf{N}|] / 2}\right)} \mathbf{A}^{\prime}\left(\sqrt{r^{2}-(i d)^{2}}\right), & \max \left(N_{R}, N_{L}\right) d<r \leq R_{t} .
\end{array}\right.
\end{aligned}
$$

where $\mathbb{E}[|\mathbf{N}|]$ is given in (??), $\mathbf{A}($.$) is given in (??) and \mathbf{A}^{\prime}($. is the derivative of $\mathbf{A}($.$) , which is always negative.$

\section{Proof: See Appendix ??}

For the NFP strategy, the distribution of the transmitterreceiver distance is stated via the following lemma:

Lemma 2: The distance $r_{N}$ between the transmitter and the receiver for the $N F P$ strategy is distributed as:

\footnotetext{
${ }^{6}$ with a slight abuse of the terminology, we denote the LT of the $p d f$ of $I_{a g g}$ as the LT of $I_{a g g}$.
} 


$$
\begin{aligned}
& f_{r_{N}}(r)=
\end{aligned}
$$

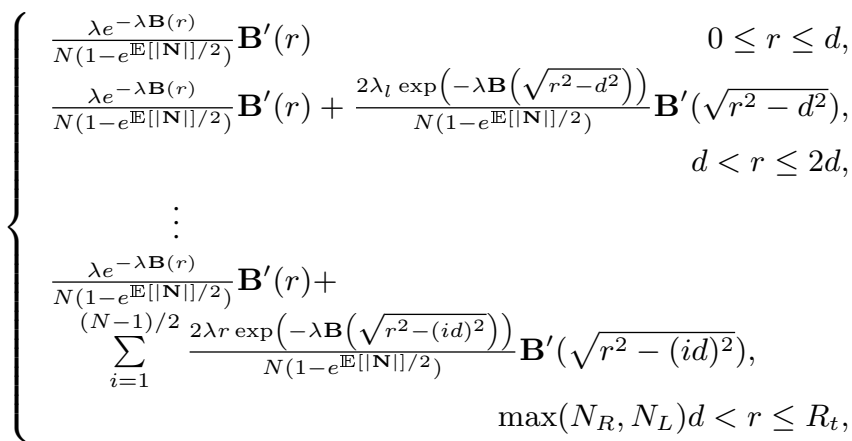

where $\mathbb{E}[|\mathbf{N}|]$ is given in (??), $\mathbf{B}($.$) is given in (??) and \mathbf{B}^{\prime}($. is the derivative of $\mathbf{B}($.$) .$

\section{Proof: See Appendix ??}

The transmitter-receiver distance distribution for the RFP is calculated via the following lemma:

Lemma 3: The distance $r_{R}$ between the transmitter and the receiver for the $R F P$ strategy is distributed as:

$$
f_{r_{R}}(r)=\left\{\begin{array}{cc}
\frac{1}{N R_{t}}, & 0 \leq r \leq d, \\
\frac{1}{N R_{t}}+\frac{2 r}{N R_{t} \sqrt{r^{2}-d^{2}}}, & d<r \leq 2 d, \\
\vdots & \\
\frac{1}{N R_{t}}+\sum_{i=1}^{(N-1) / 2} \frac{2 r}{N R_{t} \sqrt{r^{2}-(i d)^{2}}}, \\
\quad \max \left(N_{R}, N_{L}\right) d<r \leq R_{t} .
\end{array}\right.
$$

Proof: See Appendix ??

\section{B. The Intensity of Concurrent Transmitters \& LT of the Aggregate Interference}

For the sake of simple presentation, we first present the analysis of the intensity of concurrent transmitters and LT of aggregate interference for the single lane model. Then, we generalize the analysis for the multi-lane scenario.

1) Single Lane Model: The simplest highway consists of a single traffic lane and we deal with this case first. A test transmitter transmits to a receiver, located on the same lane, based on the transmission strategy selected. The remaining vehicles, which have packets to send, contend for spectrum access via the CSMA protocol, and transmitters who won the contention (i.e. have elapsed timers) transmit simultaneously. The receiver experiences interference from the set of concurrent active transmitters (i.e., the transmitters selected by the CSMA protocol) excluding the intended transmitter. The Matérn hard core point process of type II (MHCPP-II) is widely used in literature to model the set of concurrent transmitters in CSMA networks [?], [?]. The MHCPP-II captures the repulsive behavior of the point process due to the effect of the protection created around the transmitting nodes [?]. It is obtained via dependent thinning of the parent PPP, in which points are given a uniformly distributed mark $[0,1]$ and only the points which have the least mark in their neighbourhood are retained. The uniform mark corresponds to the backoff counter value chosen by each node in the CSMA protocol. Note that the interference is coming from the set $\tilde{\Phi}_{i}$ which constitutes a MHCPP. According to [?], [?], [?] the intensity of $\tilde{\Phi}_{i}$ is given by:

$$
\Lambda=\lambda \frac{1-e^{-\alpha \mathbb{E}[|\mathcal{N}|] / 2}}{\alpha \mathbb{E}[|\mathcal{N}|] / 2},
$$

where $\mathbb{E}[|\mathcal{N}|]$ is given in (??) and $1 \leq \alpha \leq 2$ is a correction factor that is used to mitigate the underestimation problem of the MHCPP-II. That is, the MHCPP-II uses $\alpha=2$ in (??) which implies a contention domain length of $2 R_{s}$ along with retaining one transmitter per contention domain. However, the MHCPP-II suffers from the intensity underestimation problem due to the role of unselected points (see [?] for details). Exploiting the natural order of points in 1-D lines, we argue that reducing the contention domain to $R_{s}$ by $\alpha=1$, as opposed to $2 R_{s}$ imposed by $\alpha=2$ for the MHCPP-II, mitigates the intensity underestimation problem.

Remark 3: The intuition behind the choice of $\alpha$ can be explained as follows; i) starting from a retained transmitter and moving in each direction along the line, the first transmitter after a void distance of $R_{s}$ contends only with the nodes in the next $R_{s}$ distance; ii) the MHCPP-II saturates at the intensity of $\frac{1}{2 R_{s}}$ which is a loose packing density. This is because if $R_{s}$ is the void region on each side of the transmitters, the intensity of concurrent transmitters should saturate at $\frac{1}{R_{s}}$. Using this order of selected points, we can deduce that in general, the contention domain is reduced by half. Thus for the multi-lane case, the average void distance between two retained points in any lane is on average greater than or equal to the sum of the forward line segments inside the circle of radius $R_{s}$. For example, in a 2-lane highway, the average void distance per lane in the saturated case is $R_{s}+\sqrt{R_{s}^{2}-d^{2}}$. Therefore, throughout the paper, we use $\alpha=1$.

Finding the LT of interference associated with a MHCPP is an open research problem because there is no known expressions of the probability generating functional for the MHCPP. Therefore, only approximate expressions for the LT are obtained by approximating the MHCPP with an equi-dense PPP existing outside the interference protection region created by the test transmitter [?]. This is formally stated as:

Assumption 3: We assume that the interfering set of vehicles $\tilde{\Phi}_{i}$ constitutes a PPP with intensity $\Lambda$ existing outside the average interference protection imposed by the CSMA protocol as shown in Fig. ??. The PPP assumption has proven to be accurate if the intensity $\Lambda$ and interference boundaries are carefully calculated [?], [?], [?].

The Laplace Transform of $I_{a g g}$ for the single lane model is given by the following lemma:

Lemma 4: The Laplace transform of the aggregate interference at a test receiver placed at the origin in the case of a single lane highway is expressed as:

$$
\begin{aligned}
\mathcal{L}_{I_{\text {agg }}}(s) & =\exp \left(-\Lambda\left(\frac{P s}{\mu}\right)^{\frac{1}{\eta}} \int_{\mathbb{R}^{1}} \frac{1}{1+t^{\eta}} d t\right), \\
& \stackrel{(\eta=2)}{=} e^{\left(-\Lambda \sqrt{\frac{P s}{\mu}} \tan ^{-1}\left(\frac{\sqrt{\mathrm{Ps} / \mu}}{\mathrm{R}_{\mathrm{S}}+\mathrm{r}}\right)-\Lambda \sqrt{\frac{\mathrm{Ps}^{\mu}}{\mu}} \tan ^{-1}\left(\frac{\sqrt{\mathrm{P}_{\mathrm{s}} / \mu}}{\mathrm{R}_{\mathrm{S}}-\mathrm{r}}\right)\right) .}
\end{aligned}
$$




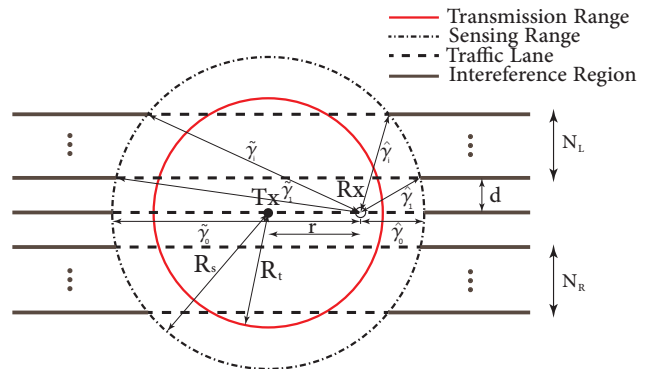

Fig. 3: Multi-lane highway with test transmitter and receiver located at the central lane with $N_{R}$ lanes on their right side and $N_{L}$ lanes on the left. The interference comes from outside the sensing range of the transmitter, marked with bold lines.

\section{Proof: See Appendix ??.}

2) Multi-lane Model: The multi-lane model illustrated in Fig. ?? is complicated and tricky to handle. We propose a three step process to deal with the interference in the multilane model. The first step is to identify concurrent transmitters via dependent thinning of the set of parallel PPPs to form a set of MHCPPs. We then approximate the MHCPP on each traffic lane with an equi-dense PPP. Finally we project the homogeneous PPPs on all the traffic lanes to nonhomogeneous PPPs on a single lane via a transformation of intensity. We use an analogy to calculate the intensity after projection and the boundaries of the PPP as shown in Fig. ?? and Fig. ??. Consider a set of uniformly spaced points on a line parallel

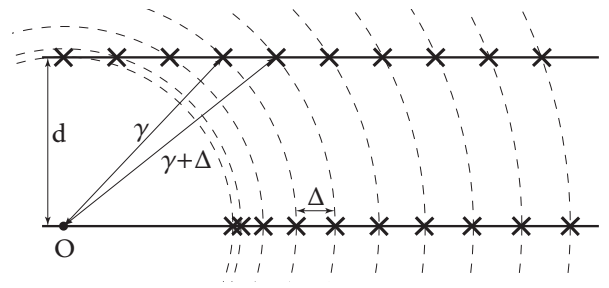

Fig. 4: Intensity Scaling during projection. A homogeneous set of points experiences a compression if mapped to the central lane with equal distance from the origin. The compression $\Delta$ is a function of the distance $\gamma$ from the origin.

to the line passing through the origin, also referred to as the central lane. From the perspective of the origin, the uniformly spaced points can be mapped to the central lane such that the distance from the origin to the points remains the same. We can observe clearly from Fig. ?? that the points experience a compression which is a function of the distance $\gamma$ from the corresponding point and the origin. The compression factor for the multi-lane scenario is given as follows:

$$
c(\gamma, i)=\frac{1}{\sqrt{\gamma^{2}+2 \sqrt{\gamma^{2}-(i d)^{2}}+1}-\gamma},
$$

where $i$ corresponds to the $i^{\text {th }}$ traffic lane away from the central lane. We can apply this principle while mapping the homogeneous poisson distributed vehicles to the central lane. Since the compression is non-linear in $\gamma$, so we obtain a nonhomogeneous PPP after the mapping operation. Therefore the intensity of the PPP used to model concurrent transmitting vehicles is given by the following lemma.

Lemma 5: The intensity of the PPP of concurrent transmitting vehicles on the $i^{\text {th }}$ traffic lane, mapped to the central lane on a multi-lane highway is given by:

$$
\tilde{\Lambda}(\gamma, i)=c(\gamma, i) \Lambda .
$$

Note that $c(\gamma, 0)=1$ and hence $\tilde{\Lambda}(\gamma, 0)=\Lambda$ i.e. the intensity of the PPP on the central lane remains unchanged under the mapping. It is worth pointing out that the intensity calculation in (??) accounts for the contention between vehicles on different lanes. This is because the expression for $\Lambda$ given in (??) contains the contention domain size $\mathbb{E}[\mathcal{N}]$ that includes vehicles in all lanes' sections covered by the sensing range as shown in Fig. 3. Using this methodology, we can extend the framework used in the single lane model to the multi-lane case. The LT of the aggregate interference for the multi-lane highway is, therefore given by the following lemma:

Lemma 6: The Laplace transform of the aggregate interference observed from a test vehicle with $N_{R}$ traffic lanes on its right and $N_{L}$ lanes on its left can be expressed as:

$$
\mathcal{L}_{I_{\text {agg }}}(s)=\mathcal{L}^{(0)}(s) \times \prod_{n=1}^{N_{R}} \mathcal{L}^{(n)}(s) \times \prod_{m=1}^{N_{L}} \mathcal{L}^{(m)}(s),
$$

where

$$
\mathcal{L}^{(i)}(s)=\exp \left(-\int_{\psi_{i} \in R^{1}}\left(\frac{\Lambda\left(\gamma_{i}, i\right)}{1+\left(\frac{\gamma_{i}}{r T^{1 / \eta}}\right)^{\eta}}\right) d \gamma_{i}\right),
$$

and $\psi_{i}$ is the interference region on the $i^{\text {th }}$ traffic lane as illustrated in Fig. ??. Thus the limits of integration are from $\hat{\gamma}_{i}$ to $\infty$ and from $-\infty$ to $\tilde{\gamma}_{i}$. The parameters $\hat{\gamma}_{i}$ and $\tilde{\gamma}_{i}$ can be expressed as follows:

$$
\begin{aligned}
\hat{\gamma}_{i} & =\sqrt{r^{2}+R_{s}^{2}+2 r \sqrt{R_{s}^{2}-(i d)^{2}}}, \\
\tilde{\gamma}_{i} & =\sqrt{r^{2}+R_{s}^{2}-2 r \sqrt{R_{s}^{2}-(i d)^{2}}}, \quad i=0,1, \ldots, \frac{N-1}{2} .
\end{aligned}
$$

\section{Proof: See Appendix ??.}

\section{Performance Analysis}

In this section we evaluate the system performance for each of the transmission strategies based on the following metrics:

1) Transmission success probability $(\mathcal{P})$

2) Normalized average forward progress (NAFP)

3) Aggregate packet progress (APP)

In the following subsections, we define these metrics along with the analysis for the different transmission strategies.

\section{A. Transmission Success Probability}

The transmission success probability, or success probability is defined as the probability that the SINR at the receiver is above a particular threshold. The general framework for evaluating the success probability has been explained in Section ??. For an $N$-lane highway with an inter-lane separation $d$, the success probability is given by the following theorem.

Theorem 1: The transmission success probability evaluated at a receiving vehicle on an $N$-lane highway with $N_{R}$ traffic 
lanes on its right and $N_{L}$ traffic lanes on its left and an interlane separation $d$ is expressed as:

$$
\begin{aligned}
& \mathcal{P}_{\chi}=\int_{0}^{R_{t}} e^{-\frac{\mu T r^{2} \kappa}{P}} e^{-\Lambda r T^{(1 / \eta)}\left(\tan ^{-1} \frac{r T^{(1 / \eta)}}{R_{s}-r}+\tan ^{-1} \frac{r T^{(1 / \eta)}}{R_{s}+r}\right)} \\
& \quad \times \prod_{i=1}^{N_{R}} \exp \left(-\int_{\psi_{i} \in \mathbb{R}^{1}}\left(\frac{\tilde{\Lambda}\left(\gamma_{i}, i\right)}{1+\left(\frac{\gamma_{i}}{r T^{1 / \eta}}\right)^{\eta}}\right) d \gamma_{i}\right) \\
& \times \prod_{j=1}^{N_{L}} \exp \left(-\int_{\psi_{j} \in \mathbb{R}^{1}}\left(\frac{\tilde{\Lambda}\left(\gamma_{j}, j\right)}{1+\left(\frac{\gamma_{j}}{r T^{1 / \eta}}\right)^{\eta}}\right) d \gamma_{j}\right) f_{r_{\chi}}(r) d r,
\end{aligned}
$$

for $\chi=\{M, N, R\}$. The interference region on the $i^{\text {th }}$ traffic lane, $\psi_{i}$ is given in Lemma??.

Proof: The proof follows by substituting the laplace transform of the aggregate interference from (??) in (??).

However the exact analytical evaluation of the expression in (??) for the multi-lane case is not possible. Note that the intractability comes from the fact that the projection of interferers leads to a non-homogeneous PPP with a complicated distance dependent factor $c(\gamma, i)$. Therefore, for tractability, we suggest two approximations to the success probability. The first is an aggressive approximation in which we assume that $\tilde{\Lambda}(\gamma, i)=\sup (c(\gamma, i)) \Lambda$. The second is a conservative approximation in which we assume that $\tilde{\Lambda}(\gamma, i)=\Lambda$. That is, the aggressive approximation neglects the intensity decay with the distance from the receiver and the conservative approximation neglects the non-uniform compression effect on the intensity of interferers due to projection. Referring to Fig. ??, the aggressive approximation means that the projected points are very close to each other experiencing maximum compression and the distance between them does not increase. On the other hand, the conservative approximation means that all projected points have the same inter-point distance as the unprojected points. Exploiting these approximations, a single integral expression for the success probability is obtained in the following two corollaries.

Corollary 1: The success probability for an $N$-lane highway is aggressively approximated by overestimating the interference by its maximum value and hence the expression in (??) reduces to:

$$
\begin{aligned}
\mathcal{P}_{\chi} \approx & \int_{0}^{R_{t}} e^{-\frac{\mu T r^{2} \kappa}{P}} e^{-\Lambda r T^{(1 / \eta)}\left(\tan ^{-1} \frac{r T^{(1 / \eta)}}{R_{s}-r}+\tan ^{-1} \frac{r T^{(1 / \eta)}}{R_{s}+r}\right)} \\
& \times \prod_{i=1}^{N_{R}} e^{-\tilde{\Lambda}\left(\hat{\gamma}_{i}, i\right) r T^{(1 / \eta)}\left(\tan ^{-1} \frac{r T^{(1 / \eta)}}{\hat{\gamma}_{i}}+\tan ^{-1} \frac{r T^{(1 / \eta)}}{\hat{\gamma}_{i}}\right)} \\
& \times \prod_{j=1}^{N_{L}} e^{-\tilde{\Lambda}\left(\hat{\gamma}_{j}, j\right) r T^{(1 / \eta)}\left(\tan ^{-1} \frac{r T^{(1 / \eta)}}{\hat{\gamma}_{j}}+\tan ^{-1} \frac{r T T^{(1 / \eta)}}{\tilde{\gamma}_{j}}\right)} f_{r_{\chi}}(r) d r
\end{aligned}
$$

for $\chi=\{M, N, R\} . \mathbb{1}_{\{.\}}$is the indicator function that is equal to 1 , if the condition in the braces is true, and 0 otherwise. The parameters $\hat{\gamma}_{i}$ and $\tilde{\gamma}_{i}$ are given in Lemma ?? and the function $\tilde{\Lambda}(\gamma, i)$ is given by (??).
Corollary 2: The success probability for an $N$-lane highway with an inter-lane separation of $d$ can be conservatively approximated by assuming the same intensity of interferers on each traffic lane i.e. $\Lambda$ and thus can be expressed as:

$$
\begin{aligned}
& \mathcal{P}_{\chi} \approx \int_{0}^{R_{t}} e^{-\frac{\mu T r^{2} \kappa}{P}} e^{-N \Lambda \sqrt{T} r \tan ^{-1} \frac{\sqrt{T r}}{\mathrm{R}_{\mathrm{s}}-\mathrm{r}}} \\
& e^{-N \Lambda \sqrt{T} r \tan ^{-1} \frac{\sqrt{T} \mathrm{r}}{\mathrm{R}_{\mathrm{s}}+\mathrm{r}}} f_{r_{\chi}}(r) d r,
\end{aligned}
$$

for $\chi=\{M, N, R\}$.

\section{B. Normalized Average Forward Progress}

The forward progress is defined as the average distance traveled by the packet towards its final destination. Note that we assume the destination is located infinitely far away and therefore the horizontal distance covered can be reasonably used as a measure of progress towards the destination. The packet progress is an important measure because it affects the number of transmissions required for transmitting a packet from the source to the destination over a multi-hop network and hence directly relates to the transmission time. The average per hop forward progress is denoted by $\overline{\mathrm{Z}}_{\chi}=\mathbb{E}\left[\mathrm{Z}_{\chi}\right]$. and is evaluated in (??), (??) and (??) for the MFR, NFP and the RFP strategies respectively.

Similar to [?], to make the average forward progress dimensionless, we normalize it by the average distance between two nearest neighbours. In this way, we transform the notion of distance (in meters) to the notion of hops (or number of nodes crossed over under a single transmission). The average distance between two nearest nodes is given by (??). Thus we can conveniently use $\bar{Z}_{\chi} \lambda$ as a measure of the normalized average forward progress (NAFP) made in a single transmission in terms of the number of nodes crossed over.

\section{Aggregate Packet Progress}

In order to have a unifying performance measure for multihop transmission we define a combined metric called the aggregate packet progress (APP). The APP captures the tradeoff between the success probability, the average forward progress, and the spatial frequency reuse efficiency. It is defined as follows:

$$
A P P_{\chi}\left(\rho_{t h}\right)=\mathcal{P}_{\chi}\left(\rho_{t h}\right) \times \Lambda\left(\rho_{t h}\right) \times \overline{\mathrm{Z}}_{\chi} .
$$

The APP is a dimensionless quantity that tells the average forward distance traveled by successfully transmitted packets per unit length in the road. Note that the success probability depends on both $\chi$ and $\rho_{t h}$, the intensity of concurrent transmitters depends only on $\rho_{t h}$ and the average forward progress depends only on $\chi$. The challenge then is to jointly select the optimal values of $\chi$ and $\rho_{t h}$ that achieve the best balance between the success probability, spatial frequency reuse efficiency and average forward progress given specific QoS requirements. The problem is formulated as follows:

$$
\begin{array}{cc}
\max _{\chi, \rho_{t h}} & A P P_{\chi}\left(\rho_{t h}\right), \\
\text { s.t. } & \frac{\lambda}{\Lambda\left(\rho_{t h}\right)}\left(\frac{\tau \delta}{\mathcal{P}_{\chi}\left(\rho_{t h}\right) \bar{Z}_{\chi}}\right) \leq \epsilon,
\end{array}
$$




$$
\begin{aligned}
& \mathbb{E}\left[\mathbf{Z}_{M}\right] \approx \frac{N}{e^{\frac{\mathbb{E}[\mathbf{N}]}{2}}-1}\left[\sum_{i=0}^{\beta} \frac{\exp \left(\lambda \sum_{j=0}^{i}\left(1+\mathbb{1}_{\left\{j>\left|N_{R}-N_{L}\right|\right\}}\right) \omega(j)\right)\left(\left(1-\lambda\left(N-\sum_{j=1}^{i}\left(1+\mathbb{1}_{\left.\left\{j>\left|N_{R}-N_{L}\right|\right\}\right)}\right) \omega(i)\right) e^{\lambda\left(N-\sum_{j=1}^{i}\left(1+\mathbb{1}\left\{j>\left|N_{R}-N_{L}\right|\right\}\right)\right) \omega(i)}\right)\right.}{\lambda\left(N-\sum_{j=1}^{i}\left(1+\mathbb{1}_{\left.\left.\left\{j>\left|N_{R}-N_{L}\right|\right\}\right)\right)^{2}}\right.\right.}\right.
\end{aligned}
$$

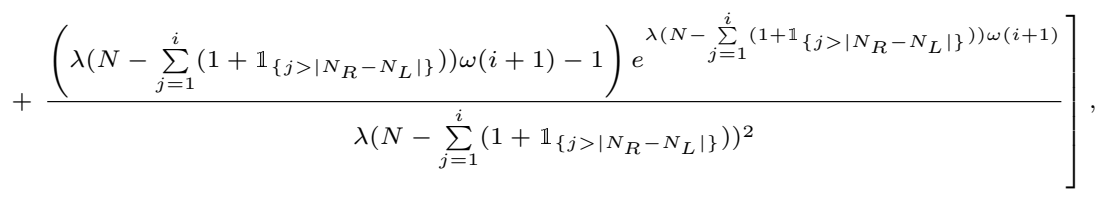

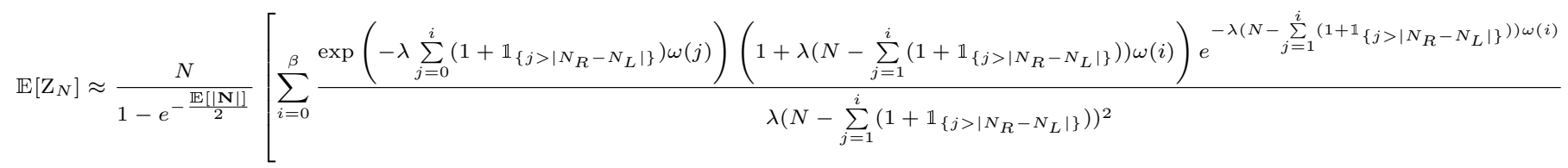

$$
\begin{aligned}
& \left.-\frac{\left.\left.\left(1+\lambda\left(N-\sum_{j=1}^{i}\left(1+\mathbb{1}_{\left\{j>\left|N_{R}-N_{L}\right|\right\}}\right)\right) \omega(i+1)\right) e^{-\lambda\left(N-\sum_{j=1}^{i}(1+\mathbb{1}\right.}\left\{j>\left|N_{R}-N_{L}\right|\right\}\right)\right) \omega(i+1)}{\lambda\left(N-\sum_{j=1}^{i}\left(1+\mathbb{1}_{\left.\left.\left\{j>\left|N_{R}-N_{L}\right|\right\}\right)\right)^{2}}\right.\right.}\right]
\end{aligned}
$$

$$
\mathbb{E}\left[\mathrm{Z}_{R}\right] \approx \frac{1}{2} \sum_{i=1}^{\beta}\left(\frac{1}{N R_{t}}+\Omega(i)\right)\left(\omega(i+1)^{2}-\omega(i)^{2}\right)
$$

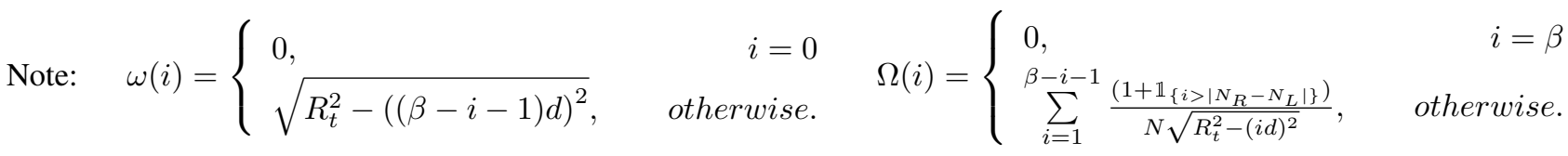

Proof: See Appendix ??

where $\delta$ is the distance from the source to destination, and $\tau$ is the duration of each time slot. The QoS constraint comes from the transmission delay or the end-to-end throughput requirements. The constraint reflects the average end-to-end transmission delay from the source to the destination and enforces it to be less than a certain threshold $\epsilon$. Note that $1 / \mathcal{P}_{\chi}$ signifies the average number of re-transmissions required to achieve successful per-hop packet transmission, $\delta / \bar{Z}_{\chi}$ is the average number of hops that are required for the packet to reach a destination located at a distance of $\delta$, and $\tau$ is the duration of each time slot. It is important to highlight that the additional delay imposed by the CSMA contention to access the spectrum can be easily incorporated to the constraint in (??) by multiplying by the factor $\frac{\lambda}{\Lambda}$, which captures the average number of time slots spent in CSMA contention to access the spectrum for a single transmission.

\section{Simplifying APPROXimations}

While the PPP approximation in Assumption ?? is mandatory for our analysis, there are some approximations which can simplify the complicated expressions in (??),(??) and (??)(??) to gain more insight. We begin with the expressions of the distance distribution between the transmitter and receiver. The distance distribution for the $M F R$ forwarding protocol given by (??) can be approximated as:

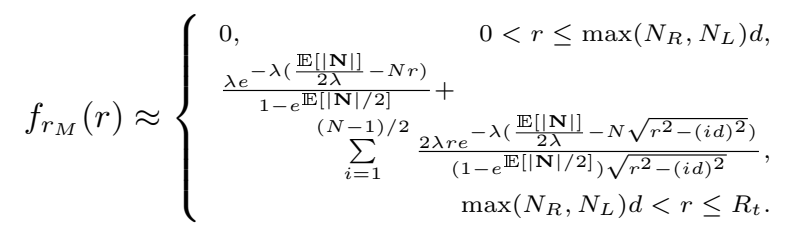

This approximation is based on the fact that the receiver, in the case of the $M F R$ forwarding strategy, is located near the edge of the transmission range with high probability and thus one part of the pdf dominates resulting in this approximation which is tight for higher intensities. Similarly for the NFP forwarding strategy, we can approximate the distance distribution given by (??) as follows:

$$
f_{r_{N}}(r) \approx \frac{N \lambda e^{-\lambda N r}}{1-e^{\mathbb{E}[|\mathbf{N}| / 2]}}, \quad 0<r \leq R_{t} .
$$

This approximation is based on the nature of the NFP strategy which dictates a receiver located very close to the transmitter, thus there is a higher probability of small transmitter receiver distances which leads to the above approximation. The approximate simplified expression for $f_{r_{\chi}}($.$) not only simplifies$ the calculation for the success probability in (??), but also 


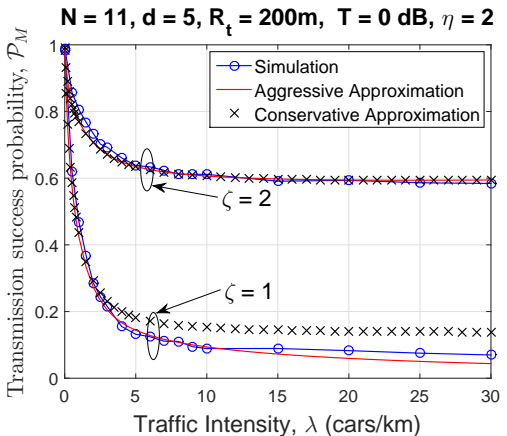

(a) Model validation for the MFR strategy

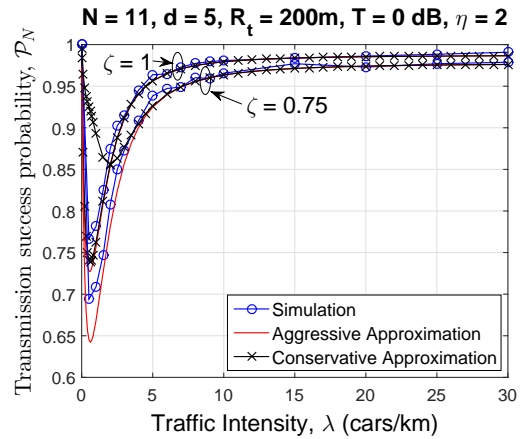

(b) Model validation for the NFP strategy

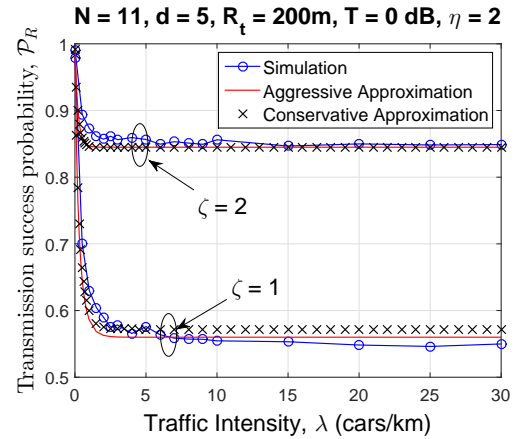

(c) Model validation for the RFP strategy

Fig. 5: Model validation for the three packet forwarding strategies. It can be observed that for small values of $\zeta$, the aggressive approximation is very close. However with increasing $\zeta$, the aggressive approximation approaches the conservative approximation. Moreover, increasing $\zeta$ increases the success probability in general.

simplifies the expressions for the expected forward progress given in (??), (??) and (??). The expected forward progress for the $M F R$ forwarding can be approximated as:

$$
\mathbb{E}\left[\mathrm{Z}_{M}\right] \approx \frac{1+\exp \left(N \lambda R_{t}\right)\left(N \lambda R_{t}-1\right)}{N \lambda(\exp (\mathbb{E}[\mathbf{N}] / 2))} .
$$

This follows from using the approximation for $\mathbf{A}(\mathrm{z})$ given by (??) under the assumption that $R_{t} \gg \max \left(N_{R}, N_{L}\right) d^{7}$ Similarly, using the approximation for $\mathbf{B}(\mathrm{z})$ given by (??) leads to the approximate average forward progress for the NFP forwarding strategy as:

$$
\mathbb{E}\left[\mathrm{Z}_{N}\right] \approx \frac{1-\exp \left(-N \lambda R_{t}\right)\left(N \lambda R_{t}+1\right)}{N \lambda} .
$$

Finally, under the same assumption, the expected forward progress for the RFP strategy can be obtained using (??) as:

$$
\mathbb{E}\left[\mathrm{Z}_{R}\right] \approx \frac{R_{t}}{2} .
$$

\section{NumERICAL RESUlTS \& DisCUSSION}

In this section, we first validate our analysis and then provide numerical results to compare the performance of the three forwarding strategies on the metrics defined in Section ?? Note that the analytical expressions validated in this section are those which are based on the approximations given in Section ??. To this end, we show that there is an optimum sensing threshold that maximizes the success probability, SFR as well as the FP under given transmission delay constraints.

\section{A. System Parameters and Model Validation}

Unless otherwise stated, the simulation parameters are selected as; transmission power $(P=1 \mathrm{~W})$, noise floor $(\kappa=-104 \mathrm{dBm})$, road length in simulation $(L=10 \mathrm{~km})$, number of traffic lanes $(N=11)$ with $N_{R}=N_{L}=5$, inter-lane distance $(d=5 \mathrm{~m})$, transmission range $\left(R_{t}=200\right.$ $\mathrm{m})$, sensing range to transmitter range ratio $(\zeta=1)$, path loss exponent $(\eta=2)$, distance to destination $(\delta=10 \mathrm{~km})$,

\footnotetext{
${ }^{7}$ This is a reasonable assumption since a vehicle on one edge of the highway should be able to communicate to the roadside base stations located near the opposite edge of the highway
}

time slot duration $(\tau=1 \mathrm{~ms})$. The vehicles are distributed on the roads as linear Poisson point processes with an intensity ranging from 0 to 30 vehicles $/ \mathrm{km}$ in each traffic lane.

The results in Fig. ??, ?? and ?? effectively validate our analysis. It can be observed that the aggressive approximation is accurate for small values of $\zeta$. However for higher values of $\zeta$, both the conservative approximation and the aggressive approximation are very close. This is because increasing $\zeta$ implies a higher sensing range which increases the interference protection around the receiver. In other words, the distance to the nearest interferer increases and since the compression factor given in (??) decays with the distance, therefore the effects of compression diminish for larger $\zeta$. Hence the aggressive approximation becomes similar to the conservative approximation, which neglects compression effects. On the other hand, for smaller values of $\zeta$, the interference protection of the receiver is low so the model becomes sensitive to the intensity and due to significant compression effects at lower $\zeta$, both approximations are different. Additionally, increasing the value of $\zeta$ improves the success probability, which is expected, due to the increased interference protection.

We now exploit our developed model to compare performance of the three packet forwarding schemes (i.e., $M F R$, $N F P$, and $R F P$ ) in terms of the normalized average forward progress. Note that in all the results, we use the aggressive approximation of the success probability since it better approximates the success probability. Figure. ?? reveals several insights to the design of forwarding schemes in CSMA coordinated vehicular networks. The figure manifests the effect of the CSMA sensing threshold on the performance of forwarding schemes. The impact of the sensing threshold on the MFR strategy is significant due to the large transmitterreceiver separation. Hence, MFR scheme requires a conservative CSMA operation. On the other hand, the effect of the sensing threshold on the NFP is not prominent due to the short transmitter-receiver separation. Hence, the NFP can sustain an aggressive CSMA operation. It is also interesting to note that even for aggressive sensing threshold, the RFP outperforms the NFP. The figure also shows that we cannot judge the performance of the forwarding scheme without looking to 


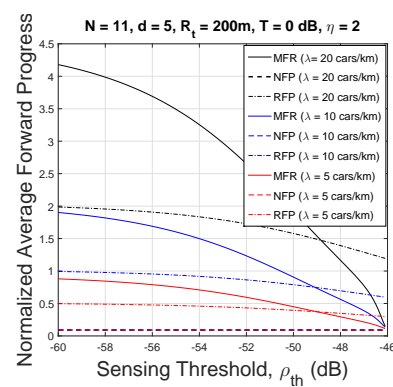

(a) NAFP against sensing range

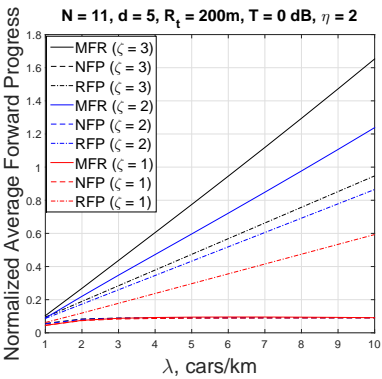

(b) NAFP against traffic intensity
Fig. 6: Normalized average forward progress against carrier sensing threshold and traffic intensity.

the sensing threshold. For instance, the $R F P$ outperforms the $M F R$ at aggressive values of the singing threshold, while the converse is true at conservative values of the sensing threshold. Finally the figure shows the effect of the traffic intensity on the forward progress. As the traffic intensity increases, the forward progress increases as the probability to find a vehicle that achieves high forward progress increases.

In Fig. ?? we plot the normalized average forward progress against different values of $\lambda$ and different values of $\zeta$ to gain further insight into the system behavior. The figure shows that for smaller values of $\zeta$, the $R F P$ strategy outperforms the $M F R$ and the $N F P$ for all traffic intensities due to the insufficient interference protection. However increasing the value of $\zeta$ increases the interference protection and leads to the outperformance of the MFR strategy. Exact critical values of $\zeta$ at which the $M F R$ outperforms the $R F P$ can be calculated from the intersecting points in Fig. ??. From Fig. ?? and Fig. ??, we observe that the NFP performs poorly in terms of the normalized average forward progress for all traffic intensities at larger values of $\zeta$.

\section{B. Maximizing aggregate packet progress}

Looking into Figs. ?? and ??, it is hard to judge the best forwarding strategy and the associated carrier sensing threshold that optimizes the tradeoff between the three performance objectives. This is because for a given success probability, the $M F R$ requires a conservative sensing threshold that may degrade the spatial frequency reuse across the road. On the other hand, the NFP can allow an aggressive sensing threshold that increases the spatial frequency reuse but results in small forward progress. Note that the key objective is to increase the number of successful parallel transmissions per unit length of road segment along with ensuring an acceptable end-to-end delay. Therefore, we define the APP with the delay constraint in (??), which is plotted in Fig. ?? for $\lambda=10$ vehicles $/ \mathrm{km}$, to capture these tradeoffs.

The APP is plotted for two different values of the SINR threshold i.e $T=0 \mathrm{~dB}$ and $T=10 \mathrm{~dB}$, number of traffic lanes i.e., $N=8\left(N_{R}=3, N_{L}=4\right)$ and $N=11$ $\left(N_{R}=5, N_{L}=5\right)$ under high and low delay tolerances. In general, it is observed that the APP is higher for $N=8$, as compared to $N=11$, due to higher success probability as
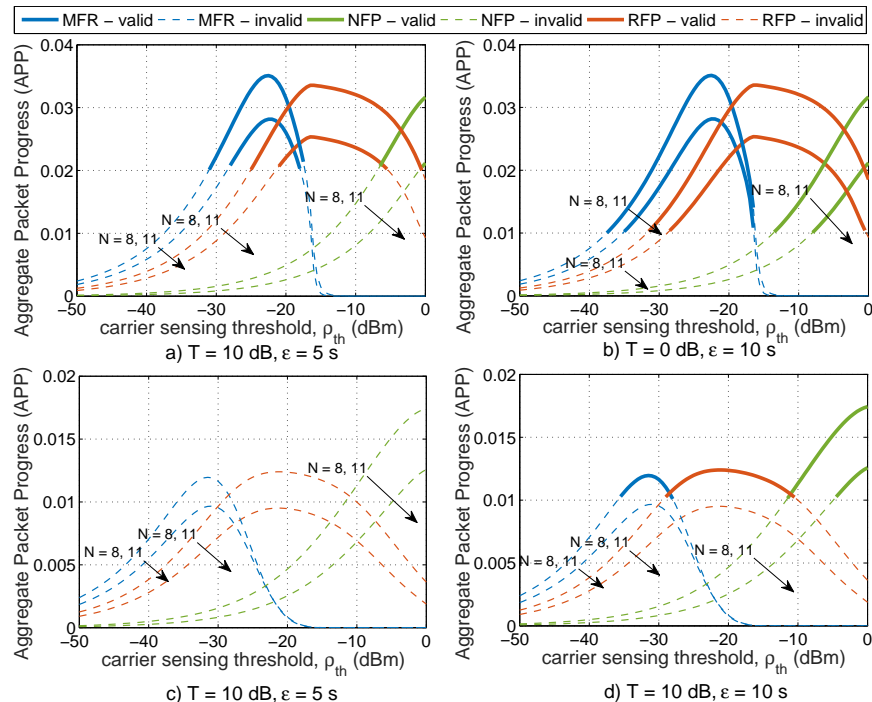

d) $\mathrm{T}=10 \mathrm{~dB}, \varepsilon=10 \mathrm{~s}$

Fig. 7: Aggregate packet progress for the $M F R, N F P$ and $R F P$ strategies against carrier sensing threshold under different SINR threshold $T$, number of traffic lanes $N$, and delay tolerance $\epsilon$ for $\lambda=10$ vehicles $/ \mathrm{km}$. Increasing $T$ allows the $N F P$ strategy to achieve a higher APP performance as compared to the $M F R$, but only at the expense of a higher $\epsilon$, which results in lower throughput.

a result of lower interference. Note that the solid line parts of the curves denote the feasible range of $\rho_{t h}$ (i.e., when the constraint in (??) is satisfied), while the dotted parts of the curves denote the infeasible range of $\rho_{t h}$. Note that the constraint is not satisfied for very low and very high values of the sensing threshold. For very low sensing threshold, the number of contending neighbours in the CSMA protocol are very large leading to high contention delay while for very high sensing threshold, the number of retransmissions required for transmission success becomes large resulting in higher transmission delay. Regardless of the number of traffic lanes $N$, it can be observed from Figs. ??(a) and ??(b) that the $M F R$, with the proper choice of $\rho_{t h}$ is preferable for lower link quality requirement (e.g., $0 \mathrm{~dB}$ ) due to the associated long forward progress. However, Figs ??(c) and ??(d) show that the NFP is the preferred forwarding scheme for high link quality requirement (e.g. $10 \mathrm{~dB}$ ), but on the expense of longer delay. This is because the MFR will require much more retransmissions to achieve the target SINR due to the distance between the transmitter and the receiver as compared with the NFP. Therefore the best forwarding strategy depends on the the required link quality per transmission. We believe that vehicular applications require lower delay e.g., safety messaging [?], so the requirements for link quality are not expected to be very aggressive and hence the $M F R$ strategy is well suited for such networks.

It is pertinent to mention here that increasing the carrier sensing threshold reduces the average protection region around a node and therefore, at sufficiently higher values of $\rho_{t h}$, the CSMA protocol starts behaving as an ALOHA. We can observe from Fig. ?? that at very high values of $\rho_{t h}$, the NFP always performs better than the $M F R$, thus verifying the result in [?], [?] that the $N F P$ is the best forwarding strategy in 
ALOHA networks.

\section{CONCLUSION \& FUTURE WORK}

A novel analytical framework for modeling CSMA coordinated Inter-vehicle Communication in Multi-Lane Highways is presented. The developed model is based on stochastic geometry and point process theory. An approximate yet accurate expressions for the intensity of concurrent transmitters and packet success probability have been obtained. We introduce the aggregate packet progress metric for multi-hop CSMA networks, which is a dimensionless quantity that shows the average forward progress traveled by successfully transmitted packets per unit length in the road. The APP is used to optimize the CSMA threshold and compare the performance of different packet forwarding schemes. By virtue of the interference coordination of the CSMA protocol, VANETs favor the MFR transmission strategy under high throughput requirements due to the associated longer forward progress. The $N F P$, however can achieve the best performance under the high SINR regime and at the expense of high transmission delay, which is not feasible. This is contradictory to the results given in [?], [?] which show that ALOHA networks always favor NFP due to the uncoordinated spectrum access. In a nutshell, the interference protection provided by the CSMA protocol enables a longer per-hop forward progress via the $M F R$ while the ALOHA protocol sacrifices the forward progress via the $N F P$ to maintain acceptable per-hop transmission success. For future work, this framework can be extended for modeling urban traffic scenarios where the traffic is restricted to crossing lanes.

\section{APPENDIX A}

PROOF OF LEMMA ??

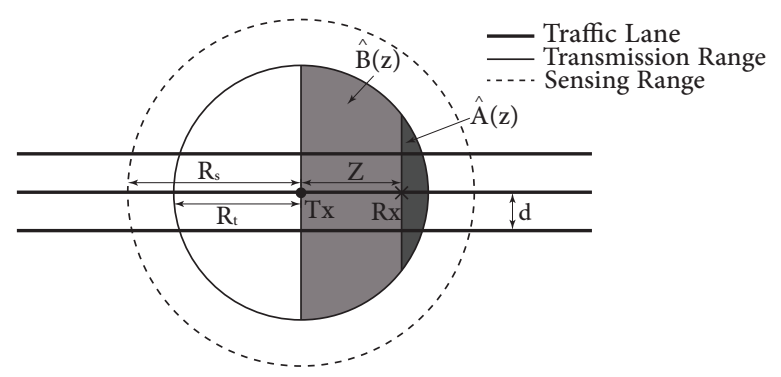

Fig. 8: Transmitter-Receiver distance for MFR strategy

Let $X+$ be the event that there exists a receiver inside the forward transmission range, illustrated by the shaded half circle in Fig.??, of the test transmitter. The probability of the event can be calculated as follows:

$$
\begin{aligned}
\mathbb{P}[X+] & =\mathbb{P}[\text { At least one vehicle in }\{\hat{\mathbf{A}}(\mathrm{z})+\hat{\mathbf{B}}(\mathrm{z})\}], \\
& =1-\mathbb{P}[\text { No vehicle in }\{\hat{\mathbf{A}}(\mathrm{z})+\hat{\mathbf{B}}(\mathrm{z})\}], \\
& =1-e^{-\mathbb{E}[|\mathbf{N}|] / 2},
\end{aligned}
$$

where $\mathbb{E}[|\mathbf{N}|] / 2$ is the average number of forward neighbours of the transmitter. Now let $Z_{\chi}$ be a random variable denoting the forward progress made by the packet in one transmission.
The subscript $\chi \in\{\mathrm{M}, \mathrm{N}, \mathrm{R}\}$ referring to the $M F R, N F P$ and $R F P$ strategy respectively. Using the methodology in [?], the conditional $\mathrm{CDF}$ of $\mathrm{Z}$ can be expressed as follows:

$$
\begin{aligned}
F_{\mathrm{Z}_{\mathrm{M}}}(\mathrm{z} \mid \mathrm{X}+) & =\mathbb{P}\left\{\mathrm{Z}_{\mathrm{M}} \leq \mathrm{z} \mid \mathrm{X}+\right\}=\frac{\mathbb{P}\{\text { No vehicle in } \hat{\mathbf{A}}(\mathrm{z}) \text { and } \mathrm{X}+\}}{\mathbb{P}\{\mathrm{X}+\}} \\
& =\frac{\mathbb{P}\{\text { No vehicle in } \hat{\mathbf{A}}(\mathrm{z}) \text { and at least one vehicle in } \hat{\mathbf{B}}(\mathrm{z})}{\mathbb{P}\{X+\}} \\
& =\frac{\mathbb{P}\{\text { No vehicle in } \hat{\mathbf{A}}(\mathrm{z})\} \mathbb{P}\{\text { At least one vehicle in } \hat{\mathbf{B}}(\mathrm{z})}{\mathbb{P}\{X+\}} \\
& =\frac{e^{-\lambda \mathbf{A}(\mathrm{z})}\left(1-e^{-\lambda \mathbf{B}(\mathrm{z})}\right)}{1-e^{-\mathbb{E}[|\mathbf{N}|] / 2}}, \\
& =\frac{e^{-\lambda \mathbf{A}(\mathrm{z})}-e^{-\mathbb{E}[|\mathbf{N}|] / 2}}{1-e^{-\mathbb{E}[|\mathbf{N}|] / 2}}, \quad 0 \leq z \leq R_{t}
\end{aligned}
$$

where $\hat{\mathbf{A}}(\mathrm{z})$ is the shaded region illustrated in Fig.?? and the length of road segment inside the shaded region, denoted by $\mathbf{A}(\mathrm{z})$, is given by:

$$
\mathbf{A}(\mathrm{z})=\left\{\begin{array}{cc}
\frac{\mathbb{E}[|\mathrm{N}|]}{2 \lambda}-N \mathrm{z}, & 0<\mathrm{z} \leq \sqrt{R_{t}^{2}-(\beta d)^{2}}, \\
\frac{\mathbb{E}[|\mathbf{N}|]}{2 \lambda}-\left(1+\mathbb{1}_{\left\{N_{R}=N_{L}\right\}}\right) \sqrt{R_{t}^{2}-(\beta d)^{2}}- \\
\left(N-\left(1+\mathbb{1}_{\left\{N_{R}=N_{L}\right\}}\right) \mathrm{z},\right. \\
\sqrt{R_{t}^{2}-(\beta d)^{2}}<\mathrm{z} \leq \sqrt{R_{t}^{2}-((\beta-1) d)^{2}}, \\
\vdots \\
\frac{\mathbb{E}[|\mathrm{N}|]}{2 \lambda}-\sum_{i=1}^{N_{R}} \sqrt{R_{t}^{2}-(i d)^{2}}-\sum_{i=1}^{N_{L}} \sqrt{R_{t}^{2}-(i d)^{2}}-\mathrm{z} . \\
\sqrt{R_{t}^{2}-d^{2}}<\mathrm{z} \leq R_{t}
\end{array}\right.
$$

where $\beta=\max \left(N_{R}, N_{L}\right)$. The conditional probability distribution of forward progress $\mathrm{Z}$ can then be evaluated by taking the derivative of (??):

$$
f_{\mathrm{Z}_{\mathrm{M}}}(\mathrm{z} \mid \mathrm{X}+)=\frac{-\lambda e^{-\lambda \mathbf{A}(\mathrm{z})}}{1-e^{-\mathbb{E}[|\mathbf{N}|] / 2}} \times \mathbf{A}^{\prime}(\mathrm{z}), \quad 0 \leq \mathrm{z} \leq R_{t},
$$

where $\mathbf{A}^{\prime}(\mathrm{z})=\frac{\mathrm{d} \mathbf{A}(\mathrm{z})}{\mathrm{dz}}$. For a test transmitter at the origin, a potential receiver can be located either on the same traffic lane or on an adjacent lane. If the receiver is on the same lane, the conditional pdf can be written as:

$$
f_{r_{M}}\left(r \mid l_{0}\right)=f_{\mathrm{Z}_{M}}(r)=\frac{-\lambda e^{-\lambda \mathbf{A}(r)}}{1-e^{-\mathbb{E}[|\mathbf{N}|] / 2}} \mathbf{A}^{\prime}(r), \quad 0 \leq r \leq R_{t},
$$

If the receiver is located on the $i^{t h}$ adjacent traffic lane, the conditional pdf $f_{r_{M}}\left(z \mid l_{i}\right)$ for $i=\left\{1, \ldots, \max \left(N_{R}, N_{L}\right)\right\}$, can be obtained using the following procedure:

$$
\begin{aligned}
r_{M}=\mathrm{z}^{2} & +(i d)^{2}, \\
F_{r_{M}}\left(r \mid l_{i}\right)= & \mathbb{P}\left(r_{M} \leq r\right)=\mathbb{P}\left(\sqrt{\mathrm{z}^{2}+(i d)^{2}} \leq r\right), \\
& =\mathbb{P}\left(\mathrm{z} \leq \sqrt{r^{2}-(i d)^{2}}\right)=F_{\mathrm{z}}\left(\sqrt{r^{2}-(i d)^{2}}\right), \\
& =\frac{\exp \left(-\lambda \mathbf{A}\left(\sqrt{r^{2}-(i d)^{2}}\right)\right)-\exp (-\mathbb{E}[|\mathbf{N}|] / 2)}{1-e^{-\mathbb{E}[|\mathbf{N}|] / 2}} .
\end{aligned}
$$

From (??), we can obtain the conditional pdf as:

$$
\begin{array}{r}
f_{r_{M}}\left(r \mid l_{i}\right)=\frac{-\lambda r \exp \left(-\lambda \mathbf{A}\left(\sqrt{r^{2}-(i d)^{2}}\right)\right)}{\left(1-e^{-\mathbb{E}[|\mathbf{N}|] / 2}\right) \sqrt{r^{2}-(i d)^{2}}} \mathbf{A}^{\prime}\left(\sqrt{r^{2}-(i d)^{2}}\right), \\
(i d) \leq r \leq R_{t} .
\end{array}
$$


Finally the complete pdf of the transmitter-receiver distance $r_{M}$ can be obtained in the form given in (??). For the case when $R_{t} \gg \beta d, \mathbf{A}(\mathbf{z})$ can be approximately expressed as:

$$
\mathbf{A}(\mathrm{z}) \approx \frac{\mathbb{E}[|\mathbf{N}|]}{2 \lambda}-N_{\mathrm{z}}, \quad 0 \leq \mathrm{z} \leq \mathrm{R}_{\mathrm{t}} .
$$

This approximate result can be very useful in evaluating approximate expressions for the complicated distance distribution of the $M F R$ strategy given in (??). It is pertinent to mention here that the approximation is also valid for $R_{t}>\beta d$. However it becomes more accurate as $R_{t} \gg \beta d$.

\section{APPENDIX B}

\section{PROOF OF LEMMA ??}

Similar to the proof in Appendix ??, we can derive the pdf for the NFP case as follows:

$$
\begin{aligned}
F_{\mathrm{Z}_{\mathrm{N}}}(\mathrm{z} \mid \mathrm{X}+) & =\frac{\mathbb{P}\{\text { No vehicle in } \hat{\mathbf{B}}(\mathrm{z})\} \mathbb{P}\{\text { At least one vehicle in } \hat{\mathbf{A}}(\mathrm{z})}{\mathbb{P}\{X+\}}, \\
& =\frac{e^{-\lambda \mathbf{B}(\mathrm{z})}-e^{-\mathbb{E}[|\mathbf{N}|] / 2}}{1-e^{-\mathbb{E}[|\mathbf{N}|] / 2}}, \quad 0 \leq z \leq R_{t},
\end{aligned}
$$

where $\mathbf{B}(\mathbf{z})$ is the length of road segment inside the shaded region $\hat{\mathbf{B}}(\mathbf{z})$ illustrated in Fig. ??, and is expressed as:

$$
\mathbf{B}(\mathrm{z})=\left\{\begin{array}{c}
N \mathrm{z}, \\
\left(1+\mathbb{1}_{\left\{N_{R}=N_{L}\right\}}\right) \sqrt{R_{t}^{2}-(\beta d)^{2}}+\left(N-\left(1+\mathbb{1}_{\left.\left.\left\{N_{R}=N_{L}\right\}\right)\right) \mathrm{z}}\right.\right. \\
\sqrt{R_{t}^{2}-(\beta d)^{2}}<\mathrm{z} \leq \sqrt{R_{t}^{2}-((\beta-1) d)^{2}}, \\
\vdots \\
\sum_{i=1}^{N_{R}} \sqrt{R_{t}^{2}-(i d)^{2}}+\sum_{i=1}^{N_{L}} \sqrt{R_{t}^{2}-(i d)^{2}}+\mathrm{z}, \\
\sqrt{R_{t}^{2}-d^{2}}<\mathrm{z} \leq R_{t},
\end{array}\right.
$$

where $\beta=\max \left(N_{R}, N_{L}\right)$. Using the same procedure as in Appendix ??, the probability distribution for the transmitterreceiver distance $r_{N}$ can be obtained in the form given in (??). For the case when $R_{t} \gg \beta d, \mathrm{~B}(\mathrm{z})$ can be approximately expressed as:

$$
\mathbf{B}(\mathrm{z}) \approx N \mathrm{z}
$$

Again, the approximation is also valid when $R_{t}>\beta d$. The approximate $p d f$ of the forward progress in the $N F P$ case is therefore given as:

$$
f_{\mathrm{Z}_{N}}(\mathrm{z}) \approx \frac{N \lambda e^{-\lambda N \mathrm{z}}}{1-e^{\mathbb{E}[|\mathbf{N}|] / 2}}, \quad 0 \leq r \leq R_{t} .
$$

The average distance between any two closest nodes, using the approximation in (??) is given as:

$$
\mathbb{E}\left[r_{N}\right]=\int_{0}^{\infty} r f_{r_{N}}(r) d r \approx \frac{1}{N \lambda} .
$$

\section{APPENDIX C}

\section{PROOF OF LEMMA ??}

For the RFP strategy, the relay node can be any of the vehicles in the forward neighbourhood of the transmitter. Similar to the proofs in Appendix ?? and Appendix ??, it can be show that the transmitter-receiver distance for the $R F P$ strategy can be given as in (??). The probability distribution of the forward progress in the case of the RFP strategy, denoted by $\mathrm{Z}_{R}$, is given as:

$$
f_{\mathrm{Z}_{R}}(z)=\left\{\begin{array}{c}
\frac{1}{N R_{t}}+\sum_{i=1}^{(N-1) / 2} \frac{2}{N \sqrt{R_{t}^{2}-(i d)^{2}}}, \\
\frac{1}{N R_{t}}+\sum_{i=1}^{(N-3) / 2} \frac{2}{N \sqrt{R_{t}^{2}-(i d)^{2}}}, \\
\sqrt{R_{t}^{2}-(\beta d)^{2}}<z \leq \sqrt{R_{t}^{2}-(\beta d)^{2}}, \\
\vdots \\
\frac{1}{N R_{t}}, \quad \sqrt{R_{t}^{2}-d^{2}}<z \leq R_{t} .
\end{array}\right.
$$

If $R_{t} \gg \beta d, f_{\mathrm{Z}_{R}}(z)$ can be approximated as:

$$
f_{\mathrm{Z}_{R}}(z) \approx \frac{1}{R_{t}}, \quad 0 \leq z \leq R_{t}
$$

\section{APPENDIX D \\ PROOF OF LEMMA ??}

Since the set of interfering vehicles is approximated by a PPP with intensity $\Lambda$, the LT of the aggregate interference can be expressed as:

$$
\begin{aligned}
\mathcal{L}_{I_{a g g}}(s) & =\mathbb{E}_{\tilde{\Phi}_{l}}\left\{e^{\left.-s I_{\text {agg }}\right\}}=\mathbb{E}_{\tilde{\Phi}_{i}}\left[\exp \left(-s \sum_{i=1}^{N} \sum_{v_{i j} \in \tilde{\Phi}_{i} \backslash v_{0}} P h_{i j}\left\|v_{i j}\right\|^{-\eta}\right)\right],\right. \\
& =\prod_{i=1}^{N} \mathbb{E}_{\tilde{\Phi}_{i}}\left[\prod_{j \in \tilde{\Phi}_{i}} \frac{\mu}{\mu+s P\left\|v_{i j}\right\|^{-\eta}}\right] \\
& =\prod_{i=1}^{N} \exp \left(-\int_{\psi_{i} \in R^{1}}\left(\frac{\tilde{\Lambda}\left(\gamma_{i}, i\right) P s \gamma_{i}^{-\eta}}{\mu+P s \gamma_{i}^{-\eta}}\right) d \gamma_{i}\right)
\end{aligned}
$$

where $\psi_{i}$ represents the interference region on the $i^{t h}$ traffic lane. Thus the limits of integration are from $\hat{\gamma}_{i}$ to $\infty$ and $-\infty$ to $\tilde{\gamma}_{i}$ as shown in Fig. ??. For the case where the intensity of the interfering PPP is constant, the expression in (??) reduces to:

$$
\mathcal{L}_{I_{\text {agg }}}(s)=\prod_{i=1}^{N} \exp \left(-\Lambda\left(\frac{P s}{\mu}\right)^{\frac{1}{\eta}} \int_{t_{i} \in \mathbb{R}^{1}} \frac{1}{1+t_{i}^{\eta}} d t_{i}\right) .
$$

Substituting (??) in (??) and putting appropriate limits of integration for the interference region proves the lemma. Note that $N=1$ in the case of Lemma ??. In Lemma ??, the product has been broken down in terms of $N_{R}$ and $N_{L}$ for model generality.

\section{APPENDIX E \\ Proof of AVERAGE ForWARd PROGRESS}

The proof of the average forward progress $Z_{\chi}$ is done by simply taking the expectation of the forward progress for each transmission strategy. The distribution of the forward progress is given in (??), (??) and (??) respectively. For notational convenience, we define the function $\omega$ which is defined as:

$$
\omega(i)=\sqrt{R_{t}^{2}-((\beta-i-1) d)^{2}} .
$$

The average forward progress for the MFR strategy is evaluated as follows: 


$$
\begin{aligned}
& \mathbb{E}\left[\mathrm{Z}_{M}\right] \approx \int_{0}^{R_{t}} z f_{Z_{M}}(z) d z=\int_{0}^{\omega(1)} \frac{z N \lambda e^{-\lambda\left(\frac{\mathbb{E}[|\mathbf{N}|]}{2 \lambda}-N z\right)}}{1-e^{-\mathbb{E}[|\mathbf{N}|] / 2}} d z+ \\
& \int_{\omega(1)}^{\omega(2)} \frac{z N \lambda}{1-e^{-\mathbb{E}[|\mathbf{N}|] / 2}} \exp \left(-\lambda\left(\frac{\mathbb{E}[|\mathbf{N}|]}{2 \lambda}-\left(1+\mathbb{1}_{\left\{1>\left|N_{R}-N_{L}\right|\right\}}\right)\right.\right. \\
&\omega(1))-\left(N-\left(1+\mathbb{1}_{\left.\left.\left.\left.\left\{1>\left|N_{R}-N_{L}\right|\right\}\right)\right) z\right)\right) d z+\ldots+}+\ldots+\sum_{i=1}^{\beta}\left(1+\mathbb{1}_{\left\{i>\left|N_{R}-N_{L}\right|\right\}}\right)\right.\right. \\
& \int_{\omega(\beta)}^{R_{t}} \frac{z N \lambda}{1-e^{-\mathbb{E}[|\mathbf{N}|] / 2}} \exp \left(-\lambda\left(\frac{\mathbb{E}[|\mathbf{N}|]}{2 \lambda}-\sum^{\beta}\right) d z\right. \\
&\omega(i)-z)) d 6
\end{aligned}
$$

Simplifying the integrals leads to the result provided in ??. In a similar manner, we can evaluate $\mathbb{E}\left[\mathrm{Z}_{N}\right]$ and $\mathbb{E}\left[\mathrm{Z}_{R}\right]$ to obtain the expressions in (??) and (??) respectively.

\section{REFERENCES}

[1] US Census Bureau, “Transportation.” Available at: http://www.census. gov/prod/2011pubs/11statab/trans.pdf?cssp=SERP 2012.

[2] National Highway Traffic Safety Administration, "Quick facts 2013." Available at: http://www-nrd.nhtsa.dot.gov/Pubs/812100.pdf

[3] T. Economist, "The cost of traffic jams." Available at: http://www.economist.com/blogs/economist-explains/2014/11/ economist-explains-1

[4] C. Jiang, Y. Chen, and K. Liu, "Data-driven optimal throughput analysis for route selection in cognitive vehicular networks," in IEEE Journal on Selected Areas in Communications, vol. 32, pp. 2149-2162, Nov. 2014.

[5] T. Willke, P. Tientrakool, and N. Maxemchuk, "A survey of inter-vehicle communication protocols and their applications," in IEEE Communications Surveys Tutorials, vol. 11, pp. 3-20, Feb. 2009.

[6] J. Kenney, "Dedicated short-range communications (DSRC) standards in the United States," in Proceedings of the IEEE, vol. 99, pp. 1162-1182, Jul. 2011.

[7] P. Nardelli, M. Kaynia, P. Cardieri, and M. Latva-aho, "Optimal transmission capacity of ad hoc networks with packet retransmissions," in IEEE Transactions on Wireless Communications,, vol. 11, pp. 27602766, Aug. 2012.

[8] T.-C. Hou and V. Li, "Transmission range control in multihop packet radio networks," in IEEE Transactions on Communications, vol. 34, pp. 38-44, Jan. 1986.

[9] P. Nardelli, P. Cardieri, and M. Latva-aho, "Efficiency of wireless networks under different hopping strategies," in IEEE Transactions on Wireless Communications, , vol. 11, pp. 15-20, Jan. 2012.

[10] S. Zaidi, M. Ghogho, D. McLernon, and A. Swami, "Achievable spatial throughput in multi-antenna cognitive underlay networks with multihop relaying," in IEEE Journal on Selected Areas in Communications, vol. 31, pp. 1543-1558, Aug. 2013.

[11] Y. Kim, F. Baccelli, and G. de Veciana, "Spatial reuse and fairness of ad hoc networks with channel-aware CSMA protocols," in IEEE Transactions on Information Theory, vol. 60, pp. 4139-4157, Jul. 2014.

[12] B. Blaszczyszyn, P. Muhlethaler, and Y. Toor, "Maximizing throughput of linear vehicular ad-hoc neworks (VANETs): A stochastic approach," in European Wireless Conference, May 2009.

[13] B. Baszczyszyn, P. Mhlethaler, and Y. Toor, "Stochastic analysis of ALOHA in vehicular ad hoc networks," in Annals of Telecommunications, vol. 68, no. 1-2, pp. 95-106, 2013.

[14] I.-H. Ho, K. Leung, and J. W. Polak, "Optimal transmission probabilities in VANETs with inhomogeneous node distribution," in IEEE 20th International Symposium on Personal, Indoor and Mobile Radio Communications (PIMRC), Tokyo, Japan, Sep. 2009.

[15] T. Nguyen, F. Baccelli, K. Zhu, S. Subramanian, and X. Wu, "A performance analysis of CSMA based broadcast protocol in VANETs," in Proc. of IEEE International Conference on Computer Communications (INFOCOM), Turin, Italy, Apr. 2013.

[16] A. Souza, A. L. Barros, A. S. Vieira, F. Roberto, and J. C. Júnior, "An adaptive mechanism for access control in VANETs," in the 10th International Conference on Networks (ICN), St. Maarten, Sweden, Jan. 2011.
[17] R. Stanica, E. Chaput, and A.-L. Beylot, "Enhancements of IEEE 802.11 p protocol for access control on a VANET control channel," in Proc. of IEEE International Conference on Communications (ICC), Kyoto, Japan, Jun. 2011.

[18] A. Giang and A. Busson, "Modeling CSMA/CA in VANET," in Analytical and stochastic modeling techniques and applications (K. AlBegain, D. Fiems, and J.-M. Vincent, eds.), vol. 7314 of Lecture Notes in Computer Science, pp. 91-105, Springer Berlin Heidelberg, 2012.

[19] V. D. Khairnar and K. Kotecha, "Performance of vehicle-to-vehicle communication using IEEE $802.11 \mathrm{p}$ in vehicular ad-hoc network environment," CoRR, vol. abs/1304.3357, 2013.

[20] Y. Yao, L. Rao, and X. Liu, "Performance and reliability analysis of IEEE $802.11 \mathrm{p}$ safety communication in a highway environment," in IEEE Transactions on Vehicular Technology, vol. 62, pp. 4198-4212, Nov. 2013.

[21] M. J. Farooq, H. ElSawy, and M. S. Alouini, "Modeling inter-vehicle communication in multi-lane highways: A stochastic geometry approach," in Proc. of IEEE Vehicular Technology Conference (VTC) Fall, Boston, USA, Sep. 2015.

[22] S. Eichler, "Performance evaluation of the IEEE 802.11p WAVE communication standard," in IEEE 66th Vehicular Technology Conference (VTC) Fall, Sep. 2007.

[23] Z. Tong, H. Lu, M. Haenggi, and C. Poellabauer, "A stochastic geometry approach to the modeling of IEEE $802.11 \mathrm{p}$ for vehicular ad hoc networks." Available at: http://www3.nd.edu/ mhaenggi/pubs/tvt14.pdf

[24] H. ElSawy, E. Hossain, and M. Haenggi, "Stochastic geometry for modeling, analysis, and design of multi-tier and cognitive cellular wireless networks: A survey," in IEEE Communication Surveys and Tutorials, vol. 15, pp. 996-1019, Jun. 2013.

[25] H. ElSawy and E. Hossain, "A modified hard core point process for analysis of random CSMA wireless networks in general fading environments," in IEEE Transactions on Communications, vol. 61, pp. 15201534, Apr. 2013.

[26] M. Haenggi, Stochastic geometry for wireless networks. Cambridge University Press, 2012.

[27] H. Nguyen, F. Baccelli, and D. Kofman, "A stochastic geometry analysis of dense IEEE 802.11 networks," in Proc. of 26th IEEE International Conference on Computer Communications (INFOCOM), Alaska, USA, May 2007.

[28] M. Kaynia, N. Jindal, and G. Oien, "Improving the performance of wireless ad hoc networks through MAC layer design," in IEEE Transactions on Wireless Communications, vol. 10, pp. 240-252, Jan. 2011.

[29] H. ElSawy, E. Hossain, and S. Camorlinga, "Characterizing random CSMA wireless networks: A stochastic geometry approach," in Proc. of IEEE International Conference on Communications (ICC), pp. 50005004, Jun. 2012.

[30] H. ElSawy and E. Hossain, "On cognitive small cells in two-tier heterogeneous networks," in Proc. of 11th Intl. Symposium on Modeling and Optimization in Mobile, Ad Hoc, and Wireless Networks (WiOpt), Tsukuba Science City, Japan, May 2013.

[31] H. Takagi and L. Kleinrock, "Optimal transmission ranges for randomly distributed packet radio terminals," in IEEE Transactions on Communications, vol. 32, pp. 246-257, Mar. 1984.

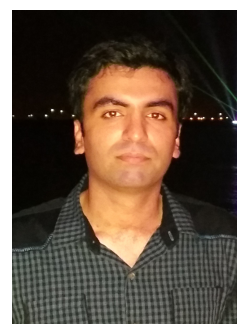

Muhammad Junaid Farooq (S'15) received the B.S. degree in Electrical Engineering from the school of electrical engineering and computer science (SEECS), National University of Sciences \& Technology (NUST), Islamabad, Pakistan in 2013 and was awarded the President's Gold medal for the best academic performance. He then received the M.S. degree in Electrical Engineering from King Abdullah University of Science and Technology (KAUST), Thuwal, Saudi Arabia, in 2015. Currently, he is a Research Assistant at the Qatar Mobility Innovations Center (QMIC), Qatar Science \& Technology Park (QSTP), Doha, Qatar. His research interests include modeling, analysis and optimization of wireless communication systems, stochastic geometry, and green communications. 


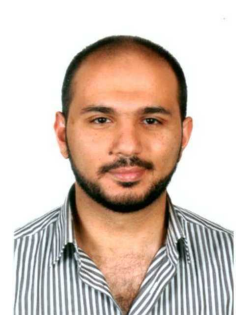

Hesham ElSawy (S'10, M'14) received the B.Sc. degree in Electrical Engineering from Assiut University, Assiut, Egypt, in 2006, the M.Sc. degree in Electrical Engineering from Arab Academy for Science and Technology, Cairo, Egypt, in 2009, and the Ph.D. degree in Electrical Engineering from the University of Manitoba, Winnipeg, MB Canada, in 2014. Currently, he is a postdoctoral fellow with the Computer, Electrical, and Mathematical Sciences and Engineering Division, King Abdullah University of Science and Technology (KAUST), Saudi Arabia, and an adjunct faculty at the school of Computer Science \& Engineering, York University, Canada. During the period of 2006-2010, he worked at the National Telecommunication Institute, Egypt, where he conducted professional training both at the national and international levels, as well as research on network planning. From 2010 to 2014, he worked with TRTech, Winnipeg, MB Canada, as a Student Researcher. For his academic excellence, he has received several academic awards, including the NSERC Industrial Postgraduate Scholarship during the period of 2010-2013, and the TRTech Graduate Students Fellowship in the period of 2010-2014. He also received the best paper award in the ICC 2015 workshop on small cells and 5G networks. He is recognized as an exemplary reviewer by the IEEE Transactions of communication. His research interests include statistical modeling of wireless networks, stochastic geometry, and queueing analysis for wireless communication networks.

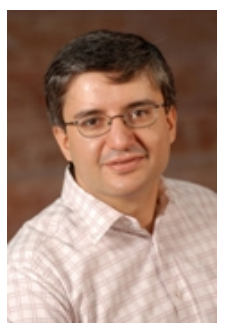

Mohamed-Slim Alouini (S'94, M'98, SM'03, F'09) was born in Tunis, Tunisia. He received the Ph.D. degree in Electrical Engineering from the California Institute of Technology (Caltech), Pasadena, CA, USA, in 1998. He served as a faculty member in the University of Minnesota, Minneapolis, MN, USA, then in the Texas A \& M University at Qatar, Education City, Doha, Qatar before joining King Abdullah University of Science and Technology (KAUST), Thuwal, Makkah Province, Saudi Arabia as a Professor of Electrical Engineering in 2009. His current research interests include the modeling, design, and performance analysis of wireless communication systems. 